\title{
Recent advances in induced pluripotent stem cell (ipsc) based therapeutics
}

\begin{abstract}
Induced Pluripotent Stem Cells (iPSC) has revolutionized the scientific field in the last decade and our approach related to stem cells based tissue engineering/regenerative medicine for disease treatment. iPSC are cells capable of differentiating into any adult/germ cells under specific condition of growth. This has led to the development of functional parenchymal cells of all the major organs till date. Particularly in the last few years we have seen a surge in development of tissue constructs on the foundation of developmental biology and tissue engineering methodology to move towards a more feasible direction of using iPSC in developing tissues/glands that can be used to replaced eteriorating/dysfunctional part of any vital organs. This review gives a brief introduction about chronological history of what led to the development of iPSCs, the mechanistic insight of pluripotency induction, molecules associated with iPSC derivation. We have also discussed about the recent advancements of use of iPSC in tissue engineering of vital organs and disease modelling, their associated limitations, and finally the advantages and limitations of iPSC technology and its future application.
\end{abstract}

Keywords: pluripotent stem cell, induduced pluripotent cells(ipsc), cell therapeutics
Volume 3 Issue 3 - 2017

\author{
Santosh Gupta,Vineeta Sharma, Rama \\ Shanker Verma \\ Department of Biotechnology, Indian Institute of Technology \\ Madras, India
}

\begin{abstract}
Correspondence: Rama Shanker Verma, Stem Cell and Molecular Biology Laboratory, Department of Biotechnology, Bhupat and Jyoti Mehta School of Biosciences, Indian Institute of Technology Madras, Chennai-600036, India, Tel +9l 442257 4109, Email vermars@iitm.ac.in
\end{abstract}

Received: July 01, 2017 | Published: September 14, 2017
Abbreviations: iPSC, induced pluripotent stem cells; ESC, embryonic stem cells; SCNT, somatic cell nuclear transfer; TFs, transcription factors; Sox2, sex determining region y-box 2; Klf4, krueppel-like factor 4; Utf1, undifferentiated embryonic cell transcription factor 1; Sall4, sal-like protein 4; hTERT, telomerase reverse transcriptase; HAT, histone acetyltransferase; CBP, creb binding protein; MSC, mesenchymal stem cells; PDAC, pancreatic cancer cell model; BMEC, brain micro vascular endothelial cells; HD, huntington disease

\section{Introduction}

The concept of creating cells that can give rise to any type of terminally differentiated cells was an interesting and unachieved feat in science until 2006 when Yamanaka group published their report of deriving induced pluripotent stem cells from mouse skin fibroblast and further used the derived iPSC to differentiate into beating cardiomyocytes. ${ }^{1}$ One year later, they reproduced the same task with human dermal fibroblast ${ }^{2}$ paving the way for use of iPSC technology in humans "personalized" regenerative therapy. However with the advent of this new technology, came a number of problems which has to be overcome to realize the journey of iPSC in human disease treatment. Induced Pluripotent Stem Cells were created as an alternative to embryonic stem cells which can give rise to any cells type. ${ }^{3}$ This was much needed as there was an ethical concern with the use of embryonic stem cell research and its use in regenerative therapy. So, iPSC technology was the new big thing in science and has seen a surge in increased number of research publication ever since 2006. Why iPSC technology was important have many substantial points, however, how much efficient and safe this technology is yet to be optimized and studied in detail before it can be explicitly used in humans. ${ }^{4}$ In this review, we have highlighted the events that created demand and ultimately led to the discovery of iPSC in a chronological manner. Further, we have briefly mentioned the mechanism of iPSC derivation and its associated factors. We have then analysed the use of iPSc in tissue engineering/regenerative medicine and disease modelling. We have also covered the advantages and limitation associated with the use of iPS cells and its future direction.

\section{What led to the development of iPSC?}

To understand the journey of iPSC, we must first understand what we had earlier, what were the limitations associated with such cells, what led to the development of alternative cell type and how much closely iPSC resembles ESC both epigenetically and by expression of pluripotent markers.

Embryonic stem cells (ESC) are derived from Inner Cell Mass of blastocyst which has unique property of pluripotency and selfrenewal. ESCs can divide indefinitely in vitro while retaining the capacity to give rise to any cell type of the adult human. This unique identity of the ESC is maintained by the pluripotency transcriptional network along with epigenetically characteristic unique histone and DNA modifications. ${ }^{5}$ ESC was considered to be a very interesting option in regenerative therapy due to its ability to give rise to any adult cell type. However, this comes with ethical concerns as derivation of functional ESC requires mature embryo and hence posed the major limitation for its application in regenerative therapy. So researchers started to find an alternative way to create ESC like cells which will have the ability to differentiate into any adult cells type. ${ }^{6}$ Three methods have been explored to this end which includes somatic cell nuclear transfer (SCNT), cell fusion and introduction of defined transcription factors, to reprogram somatic cells to pluripotency. ${ }^{7}$ Out of the three, the latter approach has been the recent and most widely studied. ${ }^{8}$ The work of John Gurdon in 1962 gave the first evidence of creating a pluripotent cell by inserting the nucleus of somatic cells of a frog into the ovum which lead to the development of whole toad. This process became to be known as somatic cell nuclear transfer (SCNT) and gave the first evidence that cells with pluripotent characteristics 
can be created in vitro. ${ }^{9}$ In 1983, Blau et al. showed that diploid human amniocytes when fused with differentiated mouse muscle cells resulted in heterokaryons expressing human muscle genes. This was major experimental evidence showing that gene expression profile of one cell type can be achieved in other cell type if the nuclear components are introduced in other cells type..$^{10}$ In 2006, Yamanaka \& Takahashi ${ }^{1}$ used the nuclear reprogramming approach where they explored the possibility of 24 nuclear factors and ultimately reaching to the point where they found that introduction of a combination of four nuclear transcription factor (Sox2, Oct4, Klf4 and c-Myc) in terminally differentiated somatic cell can induce pluripotency. ${ }^{1,2}$
Hence the story derived from this historical chronology is that the nuclear manipulation can induce pluripotency in the cells which underwent refinement over the course of time ultimately resolving the factors for pluripotency to a combination of few nuclear factors that can modulate the expression of pluripotency related genes and induce epigenetic changes to partially or completely change the memory of the somatic state of the cells to a more naive pluripotent state. Once it was established that the pluripotency can be induced by extrinsically added factors, newer approaches like small molecule inhibitors, micro RNA, transcription factors molecular forms, as well as combinatorial approaches has well been explored to induce pluripotency (Figure 1).

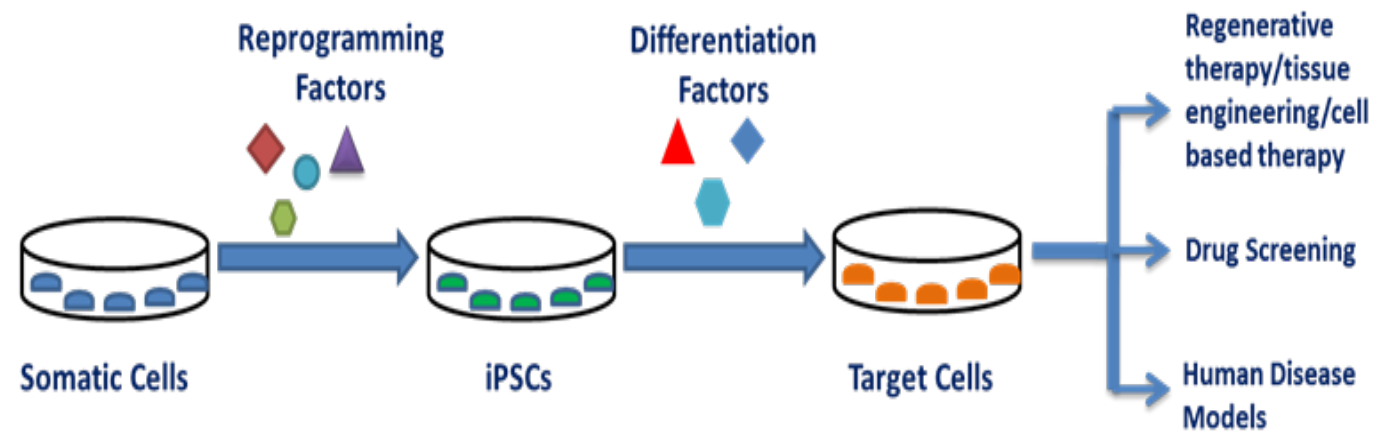

Figure I Basic approach in iPSC development and its use in Regenerative therapy/Tissue engineering.

\section{Molecular mechanism of ipsc derivation}

\section{Molecules used in pluripotency induction}

From the analysis of long list of transcription factors (TFs), Takahashi and Yamanaka concluded that a combination of just four TFs, namely octamer-binding transcription factor 3/4 (Oct3/4, O), sex determining region Y-box 2 (Sox2, S), Krueppel-like factor 4 (Klf4, $\mathrm{K}$ ), and c-Myc (M) (Yamanaka factors, OKSM), was sufficient to induced pluripotency in terminally differentiated somatic cells into a more embryonic like stage. This lead to a series of publication exploring the role of other reprogramming factors for pluripotency induction. Out of the four TF, the stochastic and temporal expression of Oct3/4 RP showed its expression to be high compared to other RFs for efficient iPSC generation.

Once the discovery of reprogramming factors for derivation of human iPSC, Thomson's team reported successful reprogramming of human fibroblast with a combination of four RFs consisting of Oct3/4 (O), Sox2 (S), Nanog (N) and Lin28 (L) (OSNL) which was different in composition from yamanaka factors (OSKM). Further, different combinations of RFs were shown to induce pluripotency like combinations consisting of five RFs (OSKMN or OSKNL) or six (OSKMNL) which were shown to have improved efficiency of iPSC generation from keratinocytes ad fibroblast. ${ }^{11}$

In addition to above combinations of RFs, a variety of other pluripotency related RFs have been tested like undifferentiated embryonic cell transcription factor 1 (Utf1), Sal-like protein 4 (Sall4), or telomerase reverse transcriptase (hTERT). Like when Utfl was over expressed together with OSKM, there was significant increase in colony formation during primary fibroblast reprogramming. iPSC were generated with Sal4 from dermal fibroblast without c-Myc over expression. Bone marrow mesenchymal stem cells and peripheral blood mononuclear cells were also reprogrammed with telomerase reverse transcriptase (hTERT) when expressed alongside OSKM and SV40 large T antigen with increased iPSC colonies. All these approaches highlights that pluripotency can be induced from factors other than yamanaka factors either in combination or in selective use of new factors that have critical role in either embryonic developmental stage or/and epigenetic landscape modifications..$^{12,13}$

\section{How pluripotency is established?}

Re-establishing pluripotency in a somatic cell is a complicated process. The most important changes include the activation of pluripotency related transcription factors and regulatory transcriptional network, re-setting the epigenetic landscape, alteration of the cell cycle. Readers are referred to the two reviews for further information which explains the mechanism in detail. ${ }^{14,15}$

Once it was shown that indeed pluripotency can be induced in a terminally differentiated somatic cells by introducing reprogramming factors, a lot of research focusing on the role of other transcription factors have been undertaken to induce pluripotency by employing a set of permutation and combination ultimately aiming to activate the pluripotency related gene network and epigenetic landscape change. A basic understanding of how pluripotency can be seen by taking consideration of the yamanaka factors that were used to induce pluripotency in mouse fibroblast. The four factors which the team narrowed down to by applying permutation and combination approach, keeping in mind the known information about each of the transcription factors, was known to perform critical role from the developmental biology perspective. In this paper, the authors explained how they concluded the combination of Oct4, Sox2, Klf4 and cMyc. Oct4 and Sox2 are important transcription factors known to be involved in pluripotency by controlling the self-renewal of embryonic stem cells. They transduced mouse fibroblast using retroviral vector expressing specific TF. Upon transduction, the cells were selected in G418 containing media (extremely high concentration, $3 \mathrm{mg} / \mathrm{ml}$ ). This was considered as iPSC cells based on their morphologically resembling ES cells.

Modulation of oct 4 results in the differentiation of stem cell and hence, it's indispensable for pluripotency. Sox 2 in association with Oct 4 control the pluripotency state in ES cell. This information helped the authors to take Oct4 and Sox 2 as one of the candidate out of the initial $24 \mathrm{TF}$. Rigorous studies lead to lead the researchers to $10 \mathrm{TF}$ 
that were required to form G418 resistant cell displaying pluripotent cell morphology. Out of the 10 factors, they removed one factor each and found that Oct4 and Sox 2 were indispensable for achieving G418 resistant morphologically distinct pluripotent cells. cMyc was another factor which they observed to be important in deriving pluripotent cells. The c-Myc protein has many downstream targets that enhance proliferation and transformation. c-Myc associates with histone acetyltransferase (HAT) complexes, including TRRAP, which is a core subunit of the TIP60 and GCN5 HAT complexes, CREB binding protein (CBP), and p300. Therefore c-Myc protein may induce global histone acetylation, thus allowing Oct $3 / 4$ and Sox 2 to bind to their specific target loci and contribute in induction of pluripotency. On the other hand, the authors found that p53 level in their iPSC was lower. It is known that Klf4 represses p53. Thus the authors suggested that Klf4 might activate nanog and other ES specific genes by repressing p53. Alternatively, they also suggested that Klf4 might act as inhibitor of c-Myc induced apoptosis through repression of p53 in their study. They also argued that K1f4 activates p $21^{\mathrm{CIP} 1}$ thereby suppressing cell proliferation. On the other hand, this anti-proliferative activity of Klf4 might be inhibited by c-Myc which suppresses the activity of $\mathrm{p} 21^{\mathrm{CIP} 1}$. Thus, the balance of Klf4 and c-Myc was essential in generation of iPSC.

Based on this study, a plethora of research articles have been published exploring the role of other genes which either along with the same combination or replacing one of the candidate from yamanaka factors (OSKM) to generate iPSCs.

\section{Pluripotency related transcription factors and regula- tory transcriptional network}

The four reprogramming factors Oct4, Sox2, Klf4 and cMyc shown to be associated with reprogramming have vital roles in early embryogenesis and ES cells. Pluripotency of ICM cells and ES cells are maintained by POU domain of transcription factor Oct4 and it is one of the most widely used reprogramming factors in iPSC development. However other factors like Nr5a2 have been used to replace Oct $3 / 4$ as a reprogramming factor. It was shown that $\mathrm{Nr} 5 \mathrm{a} 2$ binds to the promoter of Oct $3 / 4$ and Nanog and therefore activate the expression of oct3/4. This highlights the importance of pluripotency regulatory gene network and to pin point very critical molecules that can regulate important transcription factors that leads to reprogramming of cells. On the same analogy, it has also been observed that cells that expresses more OCT4 interacting proteins, such as neural stem cells and melanocytes where SOX2, a Sry-related high mobility group box transcription factor, is present, one can obtain iPS cells with higher efficiency and in a shorter time frame.

In mouse ES cells, Sox2 has been shown to work closely with Oct4 in regulating the transcription of key pluripotency genes, including Oct4, Sox 2 and Nanog. However Sox 2 is not indispensable for reprogramming if Oct $3 / 4$ is used in combination of other factors. Mouse ES cells expresses high level of can cooperate with Oct4Sox 2 complex to activate certain ES-cell-specific genes such as Lefty. Klf4 plays an important role in the pluripotency gene regulatory network by regulating the expression of Sox 2 and Nanog. c-Myc is a very well-studied oncogene and it seems to play a role of catalyst in the reprogramming process as it can significantly increase the efficiency of iPSC generation. c-Myc can recruit multiple chromatin remodeller like histone demthylase Lid and Histone acetyl-transferase GCN5. This induces the ectopically expressed transcription factors to activate their target genes more easily. The critical aspect of c-Myc is its tumorigenicity activity. Thus c-Myc mediated iPSc development requires careful scrutiny to ensure the silence of this gene. ${ }^{16}$

\section{Reorganising epigenetic landscape}

The epigenetic landscape and the DNA modification machinery are in different state between somatic cell and embryonic stage. In order to induce pluripotency or create a more embryonic like cells the chromatin and DNA modification machinery has to be reprogrammed resembling ES-cell-like state. The epigenetic profile of ES cells is characterized by demethylation of the promoter regions of key transcription factors like Oct4, Sox2, Nanog as well as bivalent chromatin modifications on developmentally important transcription factors. All these modification ensures a high level of expression of the core factors that maintain pluripotency and at the same time potentiating the cells in such a state which makes them easy to differentiate into any cell type in response to any developmental signalling factors. ${ }^{17,18}$

\section{iPSC as therapeutics}

iPSC has been used in deriving functional tissue specific cells under controlled differentiation and growth condition. The approach used in tissue engineering and regenerative medicine is based on deriving cells from healthy individual and inducing pluripotency which is further subjected to differentiation into specific cell type under specific condition. A similar approach is used for developing disease model by using cells from patients which may include any genetically inherited disease or metabolic disease. Such disease resembles the genetics and metabolic profile more closely as compared to cell lined used for studying given genetic or metabolic disorder (Figure 2). We have highlighted some of the recent research on use of iPSC in tissue engineering and disease model development in a tabular format indicating various parameters associated with pluripotency induction, scaffold type used, disease modelled, and their collective inference (Tables $1 \& 2$ ).

\section{Heart}

Cardiovascular disease has become the major cause of morbidity and leads to sudden cardiac death in young subject. Through development of human induced pluripotent stem cells has rekindle last decade but still this technology is restrained with its technical limitation. There are different commercially available differentiation kits which aids in cardiac differentiation from iPSCs. This cells however shows immature characteristics and closely resemble to foetal and embryonic derived cardiomyocytes than to mature adult cardiomyocytes. The introduction of $3 \mathrm{D}$ culture systems, electrical stimulations and applications of stretch force during differentiation leads to progress in maturation of iPSCs-cardiomyocytes. ${ }^{19}$

\section{Limitations}

These cells are single cell models and lacks complexity of cardiac tissue. iPSCs can differentiate to any of heterogeneous cardiac cells which can be obscuring its application. All said, iPSCs technology can help bringing gap between animal based models and adult cardiomyocytes. Since there has been need of hiPSCs derived CMs, several protocol have been developed for making this differentiation process effective. Earlier in 2014, it was reported that chemically defined medium consisting 3 component based medium, $\mathrm{L}$ ascorbic acid 2 phosphate and size derived recombinant human albumin along with small molecular based indicator is enough for differentiation up to 1:100 ratio of iPSCs to CMs. ${ }^{20}$ In 2016, it was reported that heparin can be used for differentiation of hiPSCs to CMs from more than 10 different hiPSCs lines. Since heparin is a Wnt modulator it helps in promoting robust and consistent $\mathrm{CM}$ production. Heparin based $\mathrm{CM}$ differentiation is easier and cost effective. ${ }^{21}$ 


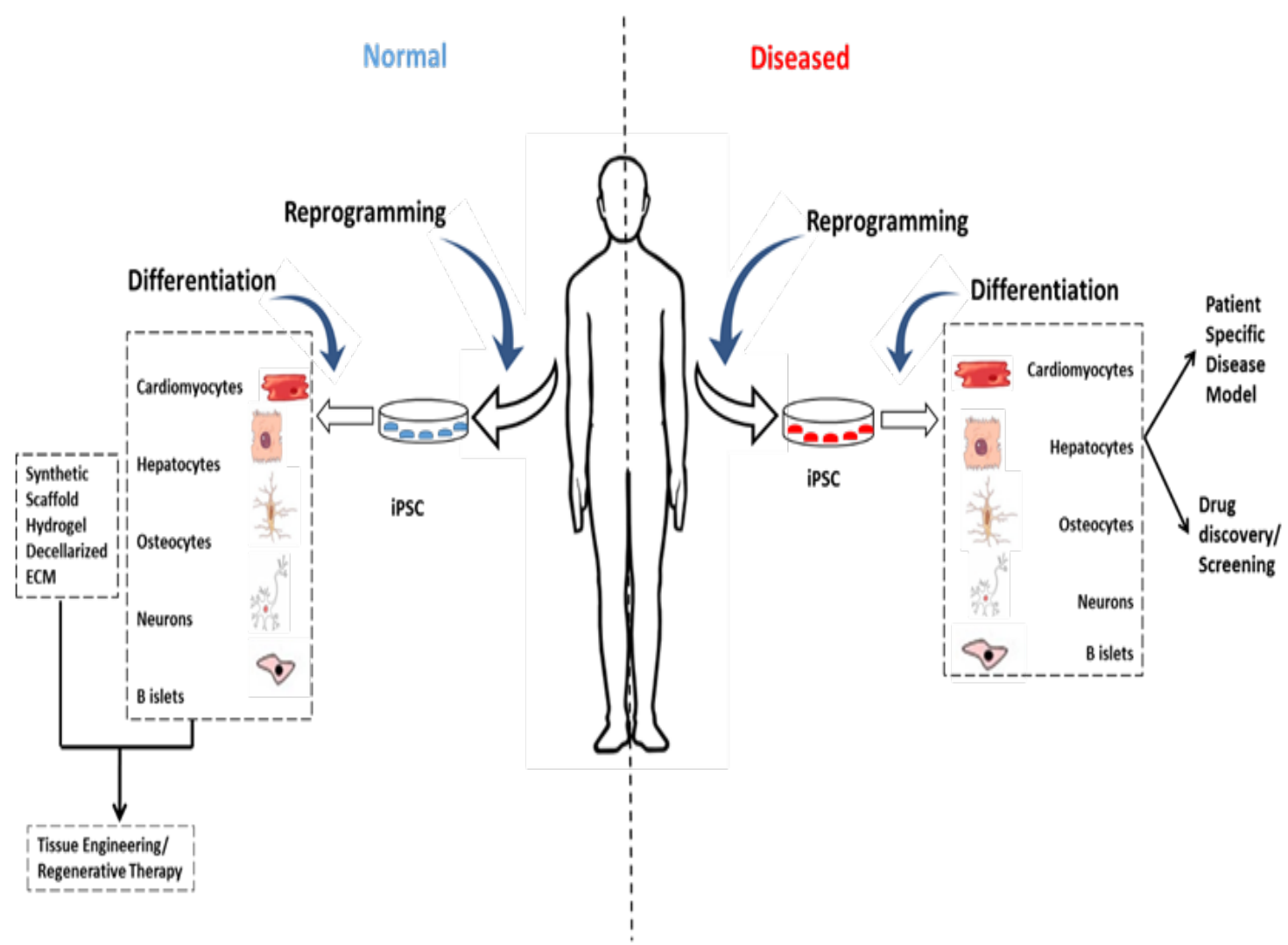

Figure 2 Schematic representation of the use of normal human derived iPSC in tissue engineering/Regenerative therapy and disease modelling from patient derived iPSC.

Table I iPSC in tissue engineering/regenerative therapy

\begin{tabular}{|c|c|c|c|c|c|c|}
\hline Cell line & $\begin{array}{l}\text { Reprogramming } \\
\text { factors }\end{array}$ & $\begin{array}{l}\text { Differentiation } \\
\text { factor }\end{array}$ & Scaffold & $\begin{array}{l}\text { Target cell } \\
\text { type }\end{array}$ & Outcome & Reference \\
\hline $\begin{array}{l}\text { BJ-RiPS-I.I } \\
\text { cell line }\end{array}$ & $\begin{array}{l}\text { KIf4, c-Myc, Oct4, } \\
\text { Sox2, Lin28 }\end{array}$ & $\begin{array}{l}\text { CHIR9902I } \\
\text { IWP-4 }\end{array}$ & $\begin{array}{l}\text { Decellularized } \\
\text { heart cardiac } \\
\text { ECM }\end{array}$ & Cardiomyocytes & $\begin{array}{l}\text { Engineered heart using iPSC developed } \\
\text { force-generating human myocardial } \\
\text { tissue and showed electrical } \\
\text { conductivity, left ventricular pressure } \\
\text { development, and metabolic function }\end{array}$ & 31 \\
\hline $\begin{array}{l}\text { C57BL/6- } \\
\text { Tg(UBC- } \\
\text { GFP)30Scha/J }\end{array}$ & $\begin{array}{l}\text { Oct } 4 \text {, Sox } 2, \text { Klf4, } \\
\text { c-Myc, }\end{array}$ & $\begin{array}{l}\text { Progesterone } \\
\text { Putrescine } \\
\text { Laminin } \\
\text { Nicotinamide } \\
\text { TS B27 media } \\
\text { supplement }\end{array}$ & $\begin{array}{l}\text { Decellularized } \\
\text { Pancreatic } \\
\text { Scaffolds }\end{array}$ & $\beta$-like cells & $\begin{array}{l}\text { Results suggested that decellularized } \\
\text { pancreatic scaffolds could provide a } \\
\text { suitable platform for cellular activities } \\
\text { of } \beta \text {-like cells including survival and } \\
\text { insulin secretion. }\end{array}$ & 32 \\
\hline MJ hiPSC & $\begin{array}{l}\text { Oct4, Sox } 2, \text { Klf4, } \\
\text { c-Myc, }\end{array}$ & $\begin{array}{l}\text { RUNX2 } \\
\text { Recombinant } \\
\text { human soluble } \\
\text { RANK ligand }\end{array}$ & $\begin{array}{l}\text { HA-PLGA/ } \\
\text { PLLA scaffold }\end{array}$ & $\begin{array}{l}\text { Osteoblasts and } \\
\text { osteoclasts }\end{array}$ & $\begin{array}{l}\text { 3D tissue-engineered bone using } \\
\text { patient-specific iPSCs showed } \\
\text { osteoclastic and osteoblastic activities }\end{array}$ & 33 \\
\hline $\begin{array}{l}\text { hiPSC line } \\
\text { (no. 0209- } \\
\text { 00I; Sidan Sai } \\
\text { Biotechnology } \\
\text { Co., Ltd., } \\
\text { Shanghai, } \\
\text { China) }\end{array}$ & $\begin{array}{l}\text { Oct3/4, Sox2, KIf4, } \\
\text { c-Myc, Nanog, } \\
\text { Lin } 28\end{array}$ & $\begin{array}{l}\text { DMEM/FI2, I0\% } \\
\text { FBS }\end{array}$ & PLGA & Chondrocytes & $\begin{array}{l}\text { At } 6 \text { weeks, cartilage-like tissue was } \\
\text { observed The results indicate that iPSCs } \\
\text { have the potential to repair cartilage } \\
\text { defects in vivo. Therefore, iPSCs could } \\
\text { be a new cell source for cartilage defect } \\
\text { repair. }\end{array}$ & 34 \\
\hline
\end{tabular}


Table Continued...

\begin{tabular}{|c|c|c|c|c|c|c|}
\hline Cell line & $\begin{array}{l}\text { Reprogramming } \\
\text { factors }\end{array}$ & $\begin{array}{l}\text { Differentiation } \\
\text { factor }\end{array}$ & Scaffold & $\begin{array}{l}\text { Target cell } \\
\text { type }\end{array}$ & Outcome & Reference \\
\hline $\begin{array}{l}\text { Porcine ear } \\
\text { fibroblasts }\end{array}$ & $\begin{array}{l}\text { Oct4, Sox2, KIf4, } \\
\text { c-Myc }\end{array}$ & $\begin{array}{l}\text { Activin A } \\
\text { Y27362 BMP } 4 \\
\text { bFGF Porcine } \\
\text { hepatocyte } \\
\text { growth } \\
\text { factor ITS } \\
\text { Oncostatin M } \\
\text { Dexamethasone }\end{array}$ & $\begin{array}{l}\text { Decellularized } \\
\text { whole liver }\end{array}$ & Hepatocytes & $\begin{array}{l}\text { iPSC derived hepatocytes engineered } \\
\text { liver graft expressed hepatocytes } \\
\text { marker and did not rupture in a short } \\
\text { term stud. }\end{array}$ & 35 \\
\hline
\end{tabular}

Table 2 iPSC used for deriving patient specific cells for disease modeling

Cell line

\section{Reprogramming} factors
Differentiation factor
Target cell

Disease
Outcome
Reference
CNS2hiPSC7, GSDIhiPSC7 and TYRI-hiPSCI

\section{Oct4, Sox2, c-Myc and KIf4}

Activin A KOSR HGF

Dexamethasone
Hepatocytes

Tyrosinemia, glycogen storage disease, progressive familial hereditary cholestasis, and two siblings with Crigler-Najjar syndrome

Homozygous familial Hepatocytes hypercholesterolemia (hoFH)

fibroblast

HES3, H9 H9

reporter line (LMXIA-eGFP knock-in PITX3eGFP knock-in RM3.5 409B2
Human derma fibroblasts
Ventral

midbrain dopamine (vmDA) neurons
Parkinson

(1)

Ascll, Myt II, and Sox 2
Progesterone

Putrescine

Neurotrophic factor Glial cell-derived

Neurotrophic factor Ciliary neurotrophic factor

KSR SMAD inhibitors LDN193189

SB43 I542 Sonic hedgehog C25II

Purmorphamine

CHIR9902I

NBN2B27

Neurotrophic factor

Glial neurotrophic factor Transforming growth factor type $\beta 3$ Dibutyryl cAMP

CHIR9902 I Activin A KFG SANT-

Oct4, Sox2, c-Myc Retinoic acid LDNKIf4 Neurotrophin-3, and
I93189 FGFI0

Indolactam $\mathrm{V}$ SB43 I542 Glucose
Neurons

Traumatic brain injury (TBI)

Patient-specific functiona neurons for cell therapy
This article confirmed the proof-of-priciple study demonstrating specific hiPSC cell lines

Effectiveness of using iPSCs to screen for potential treatments for inborn errors of hepatic metabolism development of iver diase
Demonstrated improved engraftment outcomes and restoration of motor 39 deficits in parkinsonian rat model
Pancreatic organoid resembling acinar and ductal progeny
Pancreatic facets of cystic fibrosis
Proof-of-concept screen in $\mathrm{CF}$ pancreatic organoid using a set of CFTR correctors and activators, and established an mRNA-mediated gene organoid therapy approach in CF 


\section{Liver}

The translation studies of wild hepatocytes in humans has not been successful attributing to limited source of high quality cells and continued requirement of lifelong immunosuppression. The use of patient specific hiPSC derived hepatocytes can be useful for treating inherited liver disorders, correcting the underlying genetic abnormality would be required. The proposed pathway can be followed as Patient derived hiPSC $>$ gene editing $>$ hiPSCs with corrected genes $>$ directed differentiation $>$ treatment of patient. Using gene editing and hiPSCs, it can be possible to treat many monogenetic inherited liver disorders. ${ }^{22}$ Researches with iPSc are still under exploratory phase in liver disease treatment especially in case of Acute Liver Failure. However, Mesenchymal Stem cells (MSC) have been used extensively in this regard and our laboratory has shown that human bone marrow derived mesenchymal stem cells can be trans differentiated into hepatocytes on a three dimensional fibrous scaffold. Such approach involves specifying the MSC into a more embryonic state and then differentiating the specified cells into hepatic lineage. ${ }^{23}$

Limitations: The biggest limitation of using this technology is risk of cancer, where if the undifferentiated pluripotent stem cells somehow contaminate the transplanted hepatocyte forming teratoma. ${ }^{22}$

\section{Lungs}

iPSCs have been used to recellularizing the decellularized lungs to generate different progenitor cells like lungs and airway. But differentiation of iPSCs is a time consuming process. So scientist have introduced iPSCs progenitor cells to recellularize the scaffold and it was seen that cells took 10days to differentiate to proximal and distal airway epithelial cells. There have been studies regarding regeneration of rat and human lungs using type 1 and type 2 alveolar epithelial derived from hiPSCs. Translational research of animal model has failed due to difference at molecular level between species. iPSCs derived respiratory epithelial cells, vascular endothelial cells and vascular smooth muscle cells is used for studying the development and vascular modelling. ${ }^{24}$ Patient derived iPSCs disease model can be used as personalized medicine tool for drug discovery testing. ${ }^{25}$

\section{Pancreas}

iPSCs have been used to create early pancreatic cancer cell model (PDAC) which provides insights about disease progression. This would help in discovering intrinsic precursors and secreted protein biomarkers. $^{26}$

\section{Brain}

Neurological disorder have been challenging to study in laboratory because of the lack of predictive preclinical model. Animal model is useful in elucidating disease mechanism but lacks track record when it comes to translation research. Patient specific iPSCs derived cell model provide insights in studying the effect of genetics on disease penetrance and severity. iPSC derived neuron is the only practical way to study the development and function of live human neurons. The animal model and even closely related species varies in their electro-physiological properties making the therapies effective in animal model not effective in patients. ${ }^{27}$ In recent report published in 2017 Huntington Disease (HD) iPSCs derived brain micro vascular endothelial cells have been used as disease model. Scientist found that the HD derived Brain Micro vascular Endothelial Cells (BMEC) had intrinsic impairment in angiogenic potential and drug efflux which can be rescued upon WNT inhibition. ${ }^{28}$ The better understanding of molecular basis of pluripotency, cellular reprogramming and lineage specific differentiation of iPSCs is necessary for progression in regenerative medicine.

\section{Advantages and limitations of ipsc as thera- peutics}

Every technology comes with its list of advantages and disadvantages. Here we have briefly summarized the advantages and limitations associated with iPSC technology and its use in human disease treatment and regenerative medicine..$^{29,30}$

\section{Advantages of iPSCs}

Eliminating ethical issues : Use of ESC (Embryonic stem cell) concerns with the issue regarding basic human rights, parenthood, human dignity improper incentives and health \& safety concerns of women donating eggs for the generation of embryos by in-vitro fertilization. iPSCs solves this issue by eliminating the use of embryos associated with the use of ESCs in research.

Overcoming immune rejection: iPSCs are generated from the somatic cells of one's own body thereby reducing the risk of immune rejection and hence no antigen specific secondary immune response.

Personalized medicine: Patient derived iPSCs offers opportunity for modeling disease on a patient-by-patient basis, which enables in elucidating the genomic variations among individuals that helps in understanding progression of a disease and screening of pharmacological agents suitable for an individual.

Drug development: The use of iPSCs in predicting toxicity and therapeutic responses of drugs relies on the property of iPSCs to continuously self-renew. This helps in generating libraries and the ability of iPSCs to give rise to all cell types makes them suitable to be used for predicting toxicity and possible side effects of newly developed drugs in different body cells.

Genetic manipulation: Reprogramming of somatic cells from any patients has led to the development of cell lines possessing disease specific mutations. This can be exploited to study the genomic behavior of patient specific cells by modifying the genomic components. To realize the potential of iPSC in genetic modulation and disease treatment like gene therapy, methods for efficient genetic modifications are required.

Low cost: It is estimated that for every new drug that reaches market, around 7000-10,000 compounds need to be tested during preclinical trials. Therefore strategies involving the prediction of drug toxicity and screening will increase the cost. The cost used for clinical trial could be used using iPSC which can provide toxicity details of the drug. A major component of cost in drug development goes in preclinical testing where animal models are used for assessing pharmacodynamics and pharmacokinetics parameters. As the model do not mimic the human system, use of iPSC can be useful in predicting more accurate pharmacological and toxicity profile of the drug with a higher cost-to-benefit ratio.

\section{Limitations of iPSCs}

Use of viral system for iPSC generation: Generation of iPSCs uses retroviral or lentiviral system. Genetic material inserted via retroviral 
vector may integrate randomly into the host genome causing genetic aberration.

Altered expression of reprogramming factors: Over expression of transcription factors such as Oct4, Sox2, Klf4 and c-Myc has been found to be associated with many diseased conditions such as epithelial cell dysplasia, mucinous colon carcinoma, breast cancer etc.

Restricted to monogenic phenotype: iPSCs make it possible for modeling of disease having monogenic phenotype but modeling becomes tough in case of complex disease because of uncertainty in the role of genetic and epigenetic factors.

Lower transformation efficiency - During the induction of pluripotency in somatic cells, the efficiency of induction is as low as $0.1 \%$. This leads to a lower output of desired cell type in terms of numbers from iPSC. This has to be overcome to make maximum use of pluripotent stem cells in therapeutics by increasing the efficiency of iPSC generation.

Future application of iPSC based therapeutics: The successful development of various parenchymal cells of vital organs and tissues has created a more optimistic approach in moving towards extensive use of iPSC in regenerative medicine and tissue engineering for disease treatment. With improving the efficiency of iPSC derivation, this technology can be used in development of large tissue and whole organs. The most recent use that iPSC can give is its use in development of personalized medicine and in drug testing based on pharmacogenomics principle. iPSC derived from patients can be used to create cells which can then be further used for evaluating the most effective drug for the treatment. iPSC can also be used for drug screening and newer drug development.

\section{Conclusion}

Despite overcoming the problems like limited availability, difficulty in expansion of stem cells in and immune rejection, iPSCs are certainly a step away from clinical application to its fuller extent than ES cells or mesenchymal stem cells as other critical parameters are yet to be characterized completely among which the issues related to its safety and efficacy are of paramount importance. Once this can be overcome, parameters related to the development of implants, three dimensional tissue constructs, cell laden injectable can be developed for direct use in human disease treatment. To achieve this goal, not only the understanding of basic underlying mechanism of iPSC derivation is imperative but also the differentiation of iPSC into specific cell type with cost effective method is required. From tissue engineering and regenerative point of view, a more focused approach on the behaviour of iPSCs in specific 3 dimensional environments and efficient differentiation protocol of iPSCs into specific cell type has to be established in order to create a replaceable tissue/organ for human use. ${ }^{31-40}$

\section{Acknowledgements}

The authors are thankful to Department of Biotechnology (DBT) and Indian Council of Medical Research (ICMR)for their grant support. The authors would also like to acknowledge Mr. Bhaskar Jyoti Saikia for his contribution in drafting the article.

\section{Conflict of interest}

The author declares no conflict of interest.

\section{References}

1. Takahashi K, Yamanaka S. Induction of pluripotent stem cells from mouse embryonic and adult fibroblast cultures by defined factors. Cell. 2006;126(4):663-676.

2. Takahashi K, Tanabe K, Ohnuki M, et al. Induction of pluripotent stem cells from adult human fibroblasts by defined factors. Cell. 2007;131(5):861-872.

3. Bilic J, Izpisua Belmonte JC. Concise review: Induced pluripotent stem cells versus embryonic stem cells: close enough or yet too far apart? Stem Cells. 2012;30(1):33-41.

4. Okano H, Nakamura M, Yoshida K, et al. Steps toward safe cell therapy using induced pluripotent stem cells. Circ Res. 2013;112(3):523-533.

5. Papapetrou EP. Induced pluripotent stem cells, past and future. Science. 2016;353(6303):991-992.

6. Ilic D, Ogilvie C. Concise Review: Human Embryonic Stem CellsWhat Have We Done? What Are We Doing? Where Are We Going? Stem Cells. 2017;35(1):17-25.

7. Hanna JH, Saha K, Jaenisch R. Somatic cell reprogramming and transitions between pluripotent states: facts, hypotheses, unresolved issues. Cell. 2010;143(4):508-525.

8. Shi Y, Inoue $\mathrm{H}, \mathrm{Wu} \mathrm{JC}$, et al. Induced pluripotent stem cell technology: a decade of progress. Nat Rev Drug Discov. 2017;16(2):115-130.

9. Gurdon JB. The developmental capacity of nuclei taken from intestinal epithelium cells of feeding tadpoles. J Embryol Exp Morphol. 1962;10:622-640.

10. Blau HM, Pavlath GK, Hardeman EC, et al. Plasticity of the differentiated state. Science. 1985;230(4727):758-766.

11. Buecker C, Geijsen N. Different flavors of pluripotency, molecular mechanisms, and practical implications. Cell Stem Cell. 2010;7(5):559564.

12. Kulcenty K, Wróblewska J, Mazurek S, et al. Molecular mechanisms of induced pluripotency. Contemporary Oncology. 2015;19(1A):A22A29.

13. Kunisato A, Wakatsuki M, Kodama Y, et al. Generation of induced pluripotent stem cells by efficient reprogramming of adult bone marrow cells. Stem Cells Dev. 2010;19(2):229-238.

14. Buganim Y, Faddah DA, Jaenisch R. Mechanisms and models of somatic cell reprogramming. Nature reviews Genetics. 2013;14(6):427-439.

15. Krause MN, Sancho-Martinez I, Izpisua Belmonte JC. Understanding the molecular mechanisms of reprogramming. Biochem Biophys Res Commun. 2016;473(3):693-697.

16. Na J, Plews J, Li J, et al. Molecular mechanisms of pluripotency and reprogramming. Stem Cell Res Ther. 2010;1(4):33.

17. Singh VK, Kumar N, Kalsan M, et al. Mechanism of Induction: Induced Pluripotent Stem Cells (iPSCs). J Stem Cells. 2015;10(1):43-62.

18. Liang G, Zhang Y. Embryonic stem cell and induced pluripotent stem cell: an epigenetic perspective. Cell Research. 2013;23(1):49-69.

19. Mummery CL, Zhang J, Ng ES, et al. Differentiation of Human ES and iPS Cells to Cardiomyocytes: A Methods Overview. Circulation research. 2012;111(3):344-358

20. Burridge PW, Matsa E, Shukla P, et al. Chemically Defined and Small Molecule-Based Generation of Human Cardiomyocytes. Nature methods. 2014;11(8):855-860. 
21. Lin Y, Linask KL, Mallon B, et al. Heparin Promotes Cardiac Differentiation of Human Pluripotent Stem Cells in Chemically Defined Albumin-Free Medium, Enabling Consistent Manufacture of Cardiomyocytes. Stem Cells Transl Med. 2017;6(2):527-538.

22. Rashid ST, Alexander GJ. Induced pluripotent stem cells: from Nobel Prizes to clinical applications. J Hepatol. 2013;58(3):625-629.

23. Bishi DK, Mathapati S, Venugopal JR, et al. A Patient-Inspired Ex Vivo Liver Tissue Engineering Approach with Autologous Mesenchymal Stem Cells and Hepatogenic Serum. Adv Healthc Mater. 2016;5(9):1058-1070.

24. Song JJ, Ott HC. Bioartificial lung engineering. Am J Transplant 2012;12(2):283-288.

25. Wu SM, Hochedlinger K. Harnessing the potential of induced pluripotent stem cells for regenerative medicine. Nature cell biology. 2011;13(5):497-505.

26. Kim J, Hoffman JP, Alpaugh RK, et al. An iPS Cell Line From Human Pancreatic Ductal Adenocarcinoma Undergoes Early to Invasive Stages of Pancreatic Cancer Progression. Cell reports. 2013;3(6):2088-2099.

27. Dolmetsch R, Geschwind DH. The human brain in a dish: The promise of iPSC-derived neurons. Cell. 2011;145(6):831-834.

28. Lim RG, Quan C, Reyes-Ortiz AM, et al. Huntington's Disease iPSC-Derived Brain Microvascular Endothelial Cells Reveal WNTMediated Angiogenic and Blood-Brain Barrier Deficits. Cell Rep. 2017;19(7):1365-1377

29. Singh VK, Kalsan M, Kumar N, et al. Induced pluripotent stem cells: applications in regenerative medicine, disease modeling, and drug discovery. Frontiers in Cell and Developmental Biology. 2015;3:2.

30. Walia B, Satija N, Tripathi RP, et al. Induced pluripotent stem cells: fundamentals and applications of the reprogramming process and its ramifications on regenerative medicine. Stem Cell Rev. 2012;8(1):100-115.

31. Guyette JP, Charest JM, Mills RW, et al. Bioengineering Human Myocardium on Native Extracellular Matrix. Circulation research. 2016;118(1):56-72.
32. Wan J, Huang Y, Zhou P, Guo Y, Wu C, et al. Culture of iPSCs Derived Pancreatic $\beta$-Like Cells In Vitro Using Decellularized Pancreatic Scaffolds: A Preliminary Trial. BioMed Research International. 2017;2017:4276928.

33. Jeon $\mathrm{OH}$, Panicker LM, Lu Q, et al. Human iPSC-derived osteoblasts and osteoclasts together promote bone regeneration in 3D biomaterials. Scientific Reports. 2016;6:26761.

34. Xu X, Shi D, Liu Y, et al. In vivo repair of full-thickness cartilage defect with human iPSC-derived mesenchymal progenitor cells in a rabbit model. Experimental and Therapeutic Medicine. 2017;14(1):239-245.

35. Park KM, Hussein KH, Hong SH, et al. Decellularized Liver Extracellular Matrix as Promising Tools for Transplantable Bioengineered Liver Promotes Hepatic Lineage Commitments of Induced Pluripotent Stem Cells. Tissue Eng Part A. 2016;22(5-6):449-460.

36. Ghodsizadeh A, Taei A, Totonchi M, et al. Generation of liver diseasespecific induced pluripotent stem cells along with efficient differentiation to functional hepatocyte-like cells. Stem Cell Rev. 2010;6(4):622632.

37. Cayo MA, Mallanna SK, Di Furio F, et al. A Drug Screen using Human iPSC-Derived Hepatocyte-like Cells Reveals Cardiac Glycosides as a Potential Treatment for Hypercholesterolemia. Cell Stem Cell. 2017;20(4):478-489.e5.

38. Wang P, Zhang HL, Li W, et al. Generation of Patient-Specific Induced Neuronal Cells Using a Direct Reprogramming Strategy. Stem Cells and Development. 2014;23(1):16-23.

39. Niclis JC, Gantner CW, Alsanie WF, et al. Efficiently Specified Ventral Midbrain Dopamine Neurons from Human Pluripotent Stem Cells Under Xeno-Free Conditions Restore Motor Deficits in Parkinsonian Rodents. Stem Cells Translational Medicine. 2017;6(3):937-948.

40. Hohwieler M, Illing A, Hermann PC, et al. Human pluripotent stem cell-derived acinar/ductal organoids generate human pancreas upon orthotopic transplantation and allow disease modelling. Gut. $2017 ; 66(3): 473-486$. 